\title{
RESISTÊNCIA DE CLONES DE UMEZEIRO E CULTIVARES DE PESSEGUEIRO A Meloidogyne incognita (Nemata: Heteroderidae) ${ }^{1}$
}

\author{
NEWTON ALEX MAYER ${ }^{2}$, FERNANDO MENDES PEREIRA³, JAIME MAIA DOS SANTOS ${ }^{4}$
}

\begin{abstract}
RESUMO - O presente estudo teve por objetivo avaliar a reação dos Clones 05; 10 e 15 de umezeiro (Prunus mume Sieb. et Zucc.) e das cultivares Okinawa, Aurora-1 e Dourado-1 de pessegueiro [Prunus persica (L.) Batsch] a Meloidogyne incognita (Kofoid and White) Chitwood, em condições de casa de vegetação. As plantas foram mantidas em vasos de cerâmica contendo uma mistura de solo e areia (1:1, v/v), previamente autoclavada a $121^{\circ} \mathrm{C}$ e $1 \mathrm{kgf} . \mathrm{cm}^{-2}$ por 2 horas. Aos 60 dias após o plantio, cada planta foi inoculada com 2.000 ovos e juvenis de segundo estádio de Meloidogyne incognita. O experimento foi conduzido em delineamento inteiramente casualizado, com 6 tratamentos (genótipos) e 9 repetições. Transcorridos 116 dias após a inoculação, as plantas foram colhidas para avaliação do sistema radicular. Foi possível verificar que o número de galhas por sistema radicular, o número de ovos e juvenis por $10 \mathrm{~g}$ de raízes e por sistema radicular foi nulo ou praticamente nulo em todos os clones e nas cultivares estudadas, de forma que os respectivos fatores de reprodução foram todos inferiores a 1. Conclui-se que os Clones 05; 10 e 15 de umezeiro, assim como as cultivares Okinawa, Aurora-1 e Dourado-1 de pessegueiro são resistentes a Meloidogyne incognita.
\end{abstract}

Termos para indexação: Prunus mume (Sieb. et Zucc.), Prunus persica (L.) Batsch, frutas de caroço, porta-enxerto, nematóide-de-galha.

\section{RESISTANCE OF MUME CLONES AND PEACH TREE CULTIVARS TO Meloidogyne incognita (Nemata: Heteroderidae)}

\begin{abstract}
The present study had for objective to study the reaction of mume Clones 05, 10 and 15 (Prunus mume Sieb. et Zucc.) and 'Okinawa', 'Aurora-1' and 'Dourado-1' peach tree cultivars [Prunus persica (L.) Batsch] to Meloidogyne incognita (Kofoid and White) Chitwood, in greenhouse conditions. The plants were maintained in ceramic boxes containing a soil-sand mixture $(1: 1, \mathrm{v} / \mathrm{v})$, previously autoclaved at $121^{\circ} \mathrm{C}$ and $1 \mathrm{kgf.cm}^{-2}$ for 2 hours. On the sixtieth day after planting, each plant was inoculated with 2,000 eggs in the second stage juvenile of Meloidogyne incognita. The experiment was carried out in randomized design with 6 treatments (genotypes) and 9 repetitions. After 116 days from inoculation, plants were harvested for radicular system evaluation. It was possible to verify that galls number for radicular system, eggs and juvenile number in a $10 \mathrm{~g}$ sample, eggs and juvenile number for the whole radicular system were null or practically null in all clones and cultivars studied, so that respective reproduction factors (RF) were all lower than 1 . It was verified that mume Clones 05,10 and 15, such as 'Okinawa', 'Aurora-1' and 'Dourado-1' peach tree cultivars are resistant to Meloidogyne incognita.
\end{abstract}

Index terms: Prunus mume (Sieb. et Zucc.), Prunus persica (L.) Batsch, stone fruits, rootstock, root-knot nematode.

A fruticultura moderna e competitiva exige tecnologias que permitam a obtenção de produções elevadas, regulares e de alta qualidade, com menor uso de agrotóxicos. Nesse sentido, estudos com o objetivo de identificar fontes de resistência a pragas e doenças têm se constituído importantes contribuições, não somente para o aumento da produtividade e da qualidade final das frutas, mas também sob o ponto de vista econômico da produção. A utilização de porta-enxertos resistentes a fitonematóides continua sendo a forma mais efetiva e econômica para o controle de nematóides-de-galha (Meloidogyne spp.) em frutas de caroço e pode ser especialmente importante no estabelecimento inicial e na vida produtiva do pomar, em áreas com histórico de nematóides (Pinochet et al., 1995).

Os fitonematóides prejudicam o estabelecimento inicial no campo, o crescimento, a uniformidade do pomar, a produção de frutos e a longevidade de pessegueiros [Prunus persica (L.) Batsch] em diversas regiões persícolas do mundo (Layne, 1987). Os danos associados a Meloidogyne spp. em pessegueiro incluem a diminuição do crescimento, a perda de vigor e a desfolha precoce (Nyczepir et al., 1999). Em nível mundial, estima-se que os nematóides-de-galha, considerados como os mais importantes economicamente em frutas de caroço, causam perdas de aproximadamente 12 a $15 \%$ durante o processo produtivo (Pinochet et al., 1995). Os nematóides-de-galha têm constituído um severo problema para o pessegueiro em regiões com temperaturas amenas, tais como Califórnia, Flórida, Texas, México e Israel (Layne, 1987).

No Sul do Brasil, os porta-enxertos para pessegueiro normalmente são formados a partir de caroços de diversas cultivares de maturação tardia, obtidas nas indústrias de conservas. Esse material, resíduo do processo de industrialização, pode não atender às exigências mínimas de qualidade para um bom porta-enxerto, como a resistência a fitonematóides.

Poucos são os estudos de reação de cultivares-copa de pessegueiro aos nematóides-de-galha. Entretanto, a literatura brasileira relata suscetibilidade das cultivares Rei da Conserva, Talismã, Cristal, Biuti, Magno, Aldrighi e Capdeboscq a Meloidogyne incognita e $M$. arenaria (Menten et al., 1977; Mauch et al., 1991), sendo que as duas últimas cultivares foram, no passado, as mais recomendadas para formação de porta-enxertos. Na região Sudeste do Brasil, o porta-enxerto 'Okinawa' tem sido o mais utilizado, em função do alto vigor que imprime às copas e pela resistência aos nematóides do gênero Meloidogyne (Malo, 1967; Menten et al., 1977; Scherb et al., 1994; Fachinello et al., 2000).

Nos últimos anos, no Brasil, alguns estudos têm sido realizados com o umezeiro (Prunus mume Sieb. et Zucc.), objetivando seu uso como porta-enxerto de pessegueiro, em função da compatibilidade, rusticidade e por reduzir o vigor das plantas. Em plântulas de umezeiro, obtidas por germinação de sementes, foi verificada a resistência a Meloidogyne incognita e M. javanica (Rossi et al., 2002). A literatura internacional já havia destacado a possibilidade de o umezeiro tornarse porta-enxerto para pessegueiro, por apresentar altos níveis de resistência aos nematóides-de-galha e a Clitocybe tabescens Bres., um fungo causador de podridões nas raízes (Sherman \& Lyrene, 1983).

Na Faculdade de Ciências Agrárias e Veterinárias, Universidade Estadual Paulista (FCAV/UNESP), Câmpus de Jaboticabal-SP, foram selecionados os Clones $05 ; 10$ e 15 de umezeiro com base na qualidade das raízes e nas porcentagens de enraizamento obtidas nas quatro estações do ano, quando propagados por estacas herbáceas, em câmara

\footnotetext{
(Trabalho 022/2005). Recebido: 26/01/2005. Aceito para publicação: 09/08/2005. Apoio financeiro: FAPESP. Parte da Tese de Doutorado do primeiro autor.

${ }^{2}$ Eng. Agr., Dr., Aluno do Curso de Pós-doutorado em Agronomia da Faculdade de Ciências Agrárias e Veterinárias, Câmpus de Jaboticabal. FCAV/UNESP, Departamento de Produção Vegetal, Via de Acesso Prof. Paulo Donato Castellane Km 05, CEP 14884-900, Jaboticabal-SP. Fone: (16) 3209-2668. E-mail: mayersul@yahoo.com.br.

${ }^{3}$ Eng. Agr., Dr., Prof. Voluntário do Departamento de Produção Vegetal da FCAV/UNESP, Câmpus de Jaboticabal-SP. E-mail: fmendes@fcav.unesp.br

${ }^{4}$ Eng. Agr., Prof. Assistente Doutor do Departamento de Fitossanidade da FCAV/UNESP, Câmpus de Jaboticabal-SP. E-mail: jmsantos@fcav.unesp.br
} 
TABELA 1 - Reação de clones de umezeiro e cultivares de pessegueiro a uma população inicial de 2.000 ovos e juvenis de Meloidogyne incognita, aos 116 dias após a inoculação. Jaboticabal-SP, julho de 2002.

\begin{tabular}{|c|c|c|c|c|c|}
\hline Genótipo & $\mathrm{n}^{\mathrm{o}}$ galhas/sistema radicular & $\begin{array}{c}\mathrm{n}^{\circ} \text { de ovos e juvenis } / 10 \mathrm{~g} \\
\text { raízes }\end{array}$ & $\begin{array}{c}\mathrm{n}^{0} \text { de ovos e juvenis/sistema } \\
\text { radicular }\end{array}$ & $\begin{array}{l}\text { fator de reprodução } \\
\text { (FR) }\end{array}$ & reação \\
\hline Clone 05 & 0 & 8,33 & 79,88 & 0,0399 & $\mathrm{R}$ \\
\hline Clone 10 & 0 & 0 & 0 & 0 & $\mathrm{R}$ \\
\hline Clone 15 & 0 & 0 & 0 & 0 & $\mathrm{R}$ \\
\hline Okinawa & 0,33 & 0 & 0 & 0 & $\mathrm{R}$ \\
\hline Aurora-1 & 0,33 & 0 & 0 & 0 & $\mathrm{R}$ \\
\hline Dourado-1 & 0 & 0 & 0 & 0 & $\mathrm{R}$ \\
\hline
\end{tabular}

$\mathrm{R}=$ resistente. Observação: fator de reprodução do tomateiro cv. Santa Cruz Kada $=802,2$.

de nebulização intermitente (Mayer, 2001), sendo também comprovada a resistência dos mesmos a Meloidogyne javanica (Mayer et al., 2003). No entanto, para a continuidade do processo de seleção, é indispensável a realização de novos estudos envolvendo outras espécies de fitonematóides de importância à persicultura.

O objetivo do presente trabalho foi estudar a reação de clones de umezeiro e cultivares de pessegueiro a Meloidogyne incognita, em condições de casa de vegetação.

Os Clones 05; 10 e 15 de umezeiro e a cultivar Okinawa de pessegueiro foram propagados por meio de enraizamento de estacas herbáceas, em câmara de nebulização intermitente (Mayer et al., 2001), e plântulas das cultivares de pessegueiro Aurora-1 e Dourado-1 foram obtidas por germinação de sementes, as quais foram coletadas de frutos produzidos em pomares localizados em Taiúva (SP) e na Estação Experimental de Jundiaí (SP) (Instituto Agronômico de Campinas/APTA/ SAA), respectivamente.

Para a realização do experimento, utilizaram-se vasos de cerâmica com 10 litros de capacidade, contendo substrato constituído de uma mistura de solo e areia $(1: 1, \mathrm{v} / \mathrm{v})$, previamente autoclavada a $121^{\circ} \mathrm{C}$ e $1 \mathrm{kgf} . \mathrm{cm}^{-2}$, por 2 horas. Os vasos foram mantidos em casa de vegetação coberta com plástico e laterais teladas, pertencente ao Departamento de Fitossanidade da FCAV/UNESP, Câmpus de Jaboticabal-SP. As estacas enraizadas e as plântulas foram transplantadas para os vasos contendo o substrato desinfestado em dezembro de 2001, sendo irrigadas em intervalos de aproximadamente dois dias.

Raízes de algodoeiro (Gossypium hirsutum L.) infectadas com Meloidogyne incognita (Kofoid \& White) Chitwood foram obtidas junto ao Departamento de Entomologia, Fitopatologia e Zoologia Agrícola da ESALQ/USP e levadas ao Laboratório de Nematologia da FCAV/UNESP, Câmpus de Jaboticabal-SP, onde foram preparadas as configurações perineais (Taylor \& Netscher, 1974) para a confirmação da espécie (Eisenback et al., 1981). Uma suspensão de ovos e juvenis de segundo estádio foi obtida pela técnica de Hussey \& Barker (1974), cuja concentração foi ajustada para conter 200 ovos e juvenis. $\mathrm{mL}^{-1}$ por meio de diluições e utilizada como inóculo.

Aos 60 dias após o transplante para os vasos, 9 plantas de cada clone de umezeiro e das cultivares de pessegueiro foram inoculadas, individualmente, com $10 \mathrm{~mL}$ da suspensão, aplicados com pipeta automática em quatro orifícios de $3 \mathrm{~cm}$ de profundidade, eqüidistantes entre si, no substrato ao redor das plantas. Cinco plantas de tomateiro (Lycopersicon esculentum Mill) cv. Santa Cruz Kada também foram inoculadas para a comprovação da eficiência do inóculo.

Adotou-se o delineamento inteiramente casualizado com 6 tratamentos (Clones 05; 10 e 15 de umezeiro e cultivares Okinawa, Aurora-1 e Dourado-1 de pessegueiro) e 9 repetições, sendo cada parcela constituída por uma planta. O experimento foi avaliado aos 116 dias após a inoculação. Para tanto, as plantas foram desenvasadas para a avaliação do sistema radicular, ocasião em que se mensuraram as variáveis: massa de matéria fresca do sistema radicular, número de galhas por sistema radicular, número de ovos e juvenis por $10 \mathrm{~g}$ de raízes (Coolen \& D'Herde, 1972), número de ovos e juvenis por sistema radicular e fator de reprodução (FR), segundo Cook \& Evans (1987).
Os dados obtidos para as variáveis avaliadas são apresentados na Tabela 1. Não foram encontradas galhas nos clones de umezeiro estudados e, após a extração e contagem, encontraram-se 79,88 ovos e juvenis por sistema radicular apenas no Clone 05 , sendo esse valor baixo, o que resultou em um fator de reprodução de apenas 0,0399 . Desta forma, constata-se a resistência dos Clones 05; 10 e 15 de umezeiro a Meloidogyne incognita. Esses resultados concordam com Rossi et al. (2002) que, em estudo envolvendo a reação de "seedlings" de umezeiro a $M$. incognita, constataram que o índice de galhas e de massa de ovos, bem como o número de nematóides no sistema radicular e por grama de raiz foram todos iguais a zero, caracterizando a resistência do umezeiro. Sherman \& Lyrene (1983) também relataram a resistência do umezeiro aos nematóides causadores de galhas.

As raízes de tomateiro foram severamente infectadas pelo nematóide, apresentando abundante quantidade de galhas. Após extração e contagem, o valor médio para o fator de reprodução foi de 802,2 , comprovando a viabilidade do inóculo utilizado.

Das cultivares de pessegueiro avaliadas, apenas a 'Okinawa' e 'Aurora-1' apresentaram médias de 0,33 galhas por sistema radicular, com tamanho bastante reduzido e quase imperceptíveis. Entretanto, não foram encontrados ovos ou juvenis nas três cultivares de pessegueiro, o que resultou em fatores de reprodução iguais a zero. Assim, conforme terminologia de Canto-Saénz (1985), as cultivares de pessegueiro também podem ser classificadas como resistentes a $M$. incognita. As cultivares-copa Aurora-1 e Dourado-1 foram utilizadas, pressupondo-se serem suscetíveis a $M$. incognita, o que não se confirmou. Cultivares-copa de pessegueiro com resistência a fitonematóides e outras doenças e pragas de solo podem ser interessantes para o uso como produtora direta, ou seja, dispensando o uso de porta-enxertos e da enxertia, desde que sejam propagadas por enraizamento de estacas ou propagação in vitro e que apresentem satisfatória qualidade de raízes, que possam suprir adequadamente as necessidades da planta.

A cultivar Okinawa já era citada na literatura como resistente a M incognita (Malo, 1967; Menten et al., 1977; Scherb et al., 1994; Fachinello et al., 2000). Segundo Sharpe et al. (1969), a resistência de 'Okinawa' a $M$. incognita seria conferida por um caráter monofatorial dominante e, para $M$. javanica, dois ou mais genes dominantes para resistência estariam envolvidos, sendo transferíveis aos descendentes (Pinochet et al., 1995). De acordo com Malo (1967), a resistência do 'Okinawa' a M. javanica, provavelmente, esteja baseada na habilidade dessa cultivar em restringir o suprimento alimentar e, conseqüentemente, em inibir o desenvolvimento normal do nematóide. Scherb et al. (1994), estudando a reprodução de $M$. incognita, observaram percentagens de penetração inferiores a 2,51\% nas raízes de 'Okinawa' e que os juvenis não conseguiram completar o ciclo de vida, mantendo-se no segundo estádio de desenvolvimento. Segundo esses autores, o mecanismo de resistência deve envolver aspectos fisiológicos pós-penetração de juvenis de segundo estádio.

A resistência a M. javanica (Mayer et al., 2003) e M. incognita, além da facilidade de propagação por estacas herbáceas, compatibilidade com a cv. Aurora-1 e satisfatório estabelecimento inicial no campo das mudas enxertadas nos Clones 05; 10 e 15 de umezeiro, abre boas 
perspectivas para a cultura do pessegueiro, especialmente no aspecto qualidade das mudas.

Com os resultados obtidos no presente estudo, pode-se concluir que os Clones $05 ; 10$ e 15 de umezeiro, assim como as cultivares Okinawa, Aurora-1 e Dourado-1 de pessegueiro, são resistentes a Meloidogyne incognita.

\section{REFERÊNCIAS}

CANTO-SÁENZ, M. The nature of resistance to Meloidogyne incognita (Kofoid \& White, 1919) Chitwood, 1949. In: SASSER, J.N.; CARTER, C.C. An advanced treatise on Meloidogyne; biology and control. Raleigh: North Carolina State University Graphics, 1985. p.225-231.

COOK, R.; EVANS, K. Resistance and tolerance. In: BROWN, R.H.; KERRY, B.R. (Ed.) Principles and practice of nematode control in crops. New York: Academic Press, 1987. p.179-231.

COOLEN, W.A.; D'HERDE, C.J. A method for the quantitative extraction of nematodes from plant tissue. Genth: Belgium State Agricultural Research Center, 1972.77p.

EISENBACK，J.D.; HIRSCHMANN，H.; SASSER，J.N.; TRIANTAPHYLLOU,A.C. A guide to the four most common species of root-knot nematodes (Meloidogyne species) with a pictorial key. Raleigh: The Departments of Plant Pathology and Genetics of North Carolina State University and United States Agency for International Development, 1981.48p.

FACHINELLO, J.C.; SILVA, C.A.P.; SPERANDIO, C.; RODRIGUES,A.C.; STRELOW, E.Z. Resistência de porta-enxertos para pessegueiro e ameixeira aos nematóides causadores de galhas (Meloidogyne spp.). Ciência Rural, Santa Maria, v.30, n.1, p.69-72, 2000.

HUSSEY, R.S.; BARKER, K.R. A comparison of methods of collecting inocula of Meloidogyne spp. including a new technique. Plant Disease Reporter, Saint Paul, v.57, p.1025-1028, 1974.

LAYNE, R.E.C. Peach rootstocks. In: ROM, R.C.; CARLSON, R.F. Rootstocks for fruit crops. New York: J. Wiley \& Sons, 1987. 494p.

MALO, S.E. Nature of resistence of Okinawa and Nemaguard peach to the root-knot nematode Meloidogyne javanica. Proceedings of the American Society for Horticultural Science, Alexandria, v.90, p.39-46, 1967.

MAUCH, C.H.; MAUCH, N.; FINARDI, N.L. Reações de pessegueiros e da ameixeira ao nematóide das galhas Meloidogyne incognita. Nematologia Brasileira, Piracicaba, v.15, n.1, p.59-67, 1991.

MAYER, N.A. Propagação assexuada do porta-enxerto umezeiro (Prunus mume Sieb \& Zucc.) por estacas herbáceas. 2001. $109 f$.
Dissertação (Mestrado em Agronomia) - Faculdade de Ciências Agrárias e Veterinárias, Universidade Estadual Paulista, Jaboticabal, 2001.

MAYER, N.A.; PEREIRA, F.M.; NACHTIGAL, J.C. Propagação do umezeiro (Prunus mume Sieb \& Zucc.) por estaquia herbácea. Revista Brasileira de Fruticultura, Jaboticabal, v.23, n.03, p.673676, 2001.

MAYER, N.A.; PEREIRA, F.M.; SANTOS, J.M. dos. Reação de clones de umezeiro (Prunus mume Sieb. et Zucc.) e cultivares de pessegueiro a Meloidogyne javanica (Treub, 1885) Chitwood, 1949. Revista Brasileira de Fruticultura, Jaboticabal, v.25, n.1, p.181$183,2003$.

MENTEN, J.O.M.; LORDELLO, L.G.E.; CAMPO DALL'ORTO, F.A.; OJIMA, M.; RIGITANO, O. Resistência varietal de pessegueiro (Prunus persica Batsch) aos nematóides Meloidogyne incognita e M. arenaria. In: REUNIÃO DE NEMATOLOGIA, 2., 1977, Piracicaba. Trabalhos apresentados... Piracicaba: Franciscana, 1977. v.2. p.165-173.

NYCZEPIR, A.P.; BECKMAN, T.G.; REIGHARD, G.L. Reproduction and development of Meloidogyne incognita and M. javanica on Guardian peach rootstock. Journal of Nematology, Lakeland, v.31, n.3, p.334-340, 1999.

PINOCHET, J.; FELIPE, A.; ESMENJAUD, D.; SALESSES, G.; FERNÁNDES, C. Avances en la selección de patrones de frutales de hueso frente a nematodos agalladores. Fruticultura Prefesional, Barcelona, n.71, p.28-44, 1995.

ROSSI, C.E.; FERRAZ, L.C.C.B.; MONTALDI, P.T. Resistência de frutíferas de clima subtropical e temperado a Meloidogyne incognita raça 2 e M.javanica. Arquivos do Instituto Biológico, São Paulo, v.69, n.2, p.43-49, 2002.

SCHERB, C.T.; CAMPOS, V.P.; CHALFUN, N.N.J. Penetração e reprodução de Meloidogyne incognita em pessegueiro das variedades Okinawa e R-15-2. Revista Brasileira de Fruticultura, Cruz das Almas, v.16. n.1, p.134-138, 1994.

SHARPE, R.H.; HESSE, C.O.; LOWNSBERY,B.F.; PERRY, V.G.; HANSEN, C.J. Breeding peaches for root-knot nematode resistance. Journal American Society for Horticultural Science, Alexandria, n.94, p.209-212, 1969.

SHERMAN, W.B.; LYRENE, P.M. Improvement of peach rootstock resistant to root-knot nematodes. Proceedings of the Florida State for Horticultural Society, Winter Haven, v.96, p.207-208, 1983.

TAYLOR, A. L.; NETSCHER, C. An improved technique for preparing perineal patterns of Meloidogyne spp. Nematologica, Leiden, v.20, p.268-269, 1974. 